\title{
THREE SCENARIOS FOR CHANGING OF STABILITY IN THE DYNAMIC MODEL OF NERVE CONDUCTION
}

\author{
E.A. Shchepakina \\ Samara National Research University, Samara, Russia
}

\begin{abstract}
The paper deals with the specific cases of the changing of stability of slow integral manifold of singularly perturbed systems of ODE via the dynamic model of nerve conduction. It is shown that the proper choice of the additional parameters of the system allows us to construct the slow integral manifold with multiple change of its stability.
\end{abstract}

Keywords: singular perturbations, canards, delaying of the loss of stability, critical phenomena, Hindmarsh-Rose model.

Citation: Shchepakina EA. Three scenarios for changing of stability in the dynamic model of nerve conduction. CEUR Workshop Proceedings 2016; 1638: 664-673. DOI: 10.18287/1613-0073-2016-1638-664-673

\section{Introduction}

The usual assumption of the singular perturbation theory [1-3] is based on the fact, that the main functional determinant of the fast subsystem is different from zero. However, in many applications this condition is violated that implies the critical phenomena. A violation of this condition can lead to a delaying effect of loss of stability [4]. Phenomenon of the delay of the loss of stability is based on the fact that the actual escape of the phase point from the position of equilibrium, which lost its stability, does not occur immediately. Two scenarios for delaying of the loss of stability are well-known [5].

The first case corresponds to the transition of one real eigenvalue of the linearized fast subsystem through zero when the slow variables are changed. This scenario for delaying of the loss of stability in the singularly perturbed systems is associated with the canards or duck-trajectories [6-16]. In the second case a pair of complex conjugate eigenvalues passes from the left complex half-plane to the right one [17-19].

In this paper we also consider a scenario of change of stability when the real parts as well as the imaginary parts of a pair of complex conjugate eigenvalues become zero followed by the appearance of multiple zero root and then by the birth of a pair of real eigenvalues of opposite signs.

All three scenarios of change of stability are discussed via the Hindmarsh-Rose model of nerve conduction [20]. 


\section{Delay phenomenon of loss of stability in multirate systems}

Consider the system of ordinary differential equations with a small positive parameter:

$\dot{x}=X(x, y, \varepsilon), \varepsilon \dot{y}=Y(x, y, \varepsilon)$,

where $x$ and $y$ are vectors in Euclidean spaces $\mathrm{R}^{m}$ and $\mathrm{R}^{n}$, respectively, $\varepsilon$ is a small positive parameter, the vector-functions $X$ and $Y$ are sufficiently smooth and their values are $O(1)$ as $\varepsilon \rightarrow 0$. The slow and fast subsystems are represented by the first and the second equation of (1), respectively. Such systems are called the singularly perturbed systems [1-3].

A smooth surface $S$ in $\mathrm{R}^{m} \times \mathrm{R}^{n} \times \mathrm{R}$ is called an integral manifold of the system (1) if any integral curve of the system that has at least one point in common with $S$ lies entirely on $S$. Only the integral manifolds of system (1) with dimension $m$ (the dimension of the slow variable $x$ ) that can be represented as graphs of functions

$y=h(x, \varepsilon)$,

are discussed here.

We will assume that $h(x, \varepsilon)$ is a sufficiently smooth function of $\varepsilon$. Such integral manifolds are called the manifolds of slow motions.

The surface described by the degenerate equation

$Y(x, y, 0)=0$

is called a slow surface (or a slow curve when the dimension of this surface is equal to one). The slow surface can be considered as a zero-order $(\varepsilon=0)$ approximation of the slow integral manifold, i.e., $h(x, 0)=\phi(x)$, where $\phi(x)$ is an isolated root of the degenerate equation (2).

The subset of the slow surface is stable (or attractive) if the spectrum of the Jacobian matrix

$J=\frac{\partial Y}{\partial y}(x, \phi(x), 0)$

is located in the left half-plane. If there is at least one eigenvalue of the Jacobian matrix (3) with a positive real part then the subset of the slow surface is unstable (or repulsive). The subset of the slow surface given by

$\operatorname{det}\left|\frac{\partial Y}{\partial y}(x, \phi(x), 0)\right|=0$

determines a breakdown surface (or jump points in the scalar case) [1,2].

In an "-neighborhood of a stable (unstable) subset of the slow surface there exists a stable (unstable) slow integral manifold.

A slow integral manifold can change its stability in some specific cases. The mechanism of two cases of change of stability is described below. 


\section{The case of a zero root. Canards}

Consider the autonomous singularly perturbed system

$\dot{x}=X(x, y, \alpha, \varepsilon), \varepsilon \dot{y}=Y(x, y, \alpha, \varepsilon)$,

where $\varepsilon$ is small positive parameter, $\alpha$ is an additional parameter, for which an equilibrium of the fast equation becomes unstable with transition of one real eigenvalue of (3) through zero when the slow variables are changed.

For simplicity we consider the case when the variables $x$ and $y$ are scalar. The stable and unstable subsets of the slow curve are separated by the jump point(s).

The presence of the additional scalar parameter $\alpha$ provides the possibility of gluing the stable and unstable slow invariant manifolds at a jump point to form a single trajectory, the canard.

A canard is a trajectory of a singularly perturbed system of differential equations if it follows at first a stable invariant manifold, and then an unstable one. In both cases the length of the trajectory is more than infinitesimally small.

The term "canard" (or duck-trajectory) had been originally given by French mathematicians to the intermediate periodic trajectories of the van der Pol equation between the small and the large orbits due to their special shapes [6]. However, in our work a canard is a one-dimensional slow invariant manifold of variable stability [1,2].

As the simplest system with a canard we propose

$\dot{x}=1, \varepsilon \dot{y}=x y+\alpha$.

It is clear that the trajectory $y=0$ is a canard. The left part $(x<0)$ is stable and the right part $(x>0)$ is unstable. These two parts are divided by a jump point, which separates stable and unstable parts of the slow curve, at $x=0$.

\section{The case of a pair of purely imaginary roots}

Consider the slow-fast system

$\dot{x}=\varepsilon X(x, y, \varepsilon), \dot{y}=Y(x, y, \varepsilon)$,

for which a singular point of the equation of fast motions becomes unstable with transition of a pairs of eigenvalues through the imaginary axis when the slow variables are changed [4, 17-19]. It should be noted that the system (5) can be obtained from (1) by time scaling transformation.

For analytical systems the positive semi-trajectories in a certain region of phase space tend to the curves of the degenerate system having a comparable length of motions near the stable and unstable parts of the slow surface. This describes the trajectories similar to canards, which are described above.

Slow surface of the system (5) is divided into stable and unstable regions. The first one consists of a stable equilibrium of the fast subsystem and the other one consists of the unstable steady states, their common border is called as the breakdown surface. There is an open set of points on the stable part of the slow surface starting from 
which the phase curves of the slow system intersect the breakdown surface and, under changing of $x$ along the slow curve, the pair of eigenvalues of a singular point of the equation of fast motions passes through the imaginary axis with a non-zero rate. The phase point of the system (5) starting close to the stable part of the slow surface fast (during the time of $O(|\ln \varepsilon|)$ as $\varepsilon \rightarrow 0$ ) tends to the $\varepsilon$-neighborhood of the slow surface and then moves along the slow trajectory. If the system (4) is analytic, then the further movement has an interesting and unusual phenomenon - the delay. It consists in the fact that the phase point continues to move along the unstable part of the slow surface in the $\varepsilon$-neighborhood of the slow path, more time on the order $O\left(\varepsilon^{-1}\right)$ after crossing by the slow path of the stability border. And this slow path of the trajectory along the unstable part of the slow surface has a distance of order $O(1)$. Only then can happen jump, i.e., for a time of order $O(|\ln \varepsilon|)$ (slow variables are changed by a small amount of the order $O(\varepsilon|\ln \varepsilon|)$ as $\varepsilon \rightarrow 0$ ), avoiding the slow surface at a distance of the order of $O(1)$.

This situation observed is similar to the situation with canard. The canard also moves at first along the stable part of the slow surface, and then moves along the unstable part. But canards are found in two-dimensional system with an additional parameter and they are quite rare: they exist for an exponentially small range of parameter values. And canards are the result of the transition of one real eigenvalue from the left half-plane to the right one.

\section{The change of stability on the Hindmarsh-Rose}

The Hindmarsh-Rose model [20] describes the basic properties of individual neurons, the generation of spikes and a constant level potential. In this model, Kirchhoff's law is written for each ionic currents flowing through the cell membrane.

The dimensionless form of the Hindmarsh-Rose model is

$$
\left\{\begin{array}{l}
\varepsilon \dot{x}=y-a x^{3}+b x^{2}-z+I, \\
\varepsilon \dot{y}=c-d x^{2}-y, \\
\dot{z}=s(x-\alpha)-z,
\end{array}\right.
$$

where $x$ is a transmembrane neuron potential, $y$ and $z$ are the characteristics of ionic currents dynamic, $I$ is ambient current, other parameters reflect the physical features of the neurons. The typical values of the positive parameter $\varepsilon$ are small ( $\varepsilon \square 1$ ) [21]. It should be noted that the system (6) has the type of (4) with scalar slow variable and two-dimensional fast variable.

Slow curve of system (7) is described by:

$$
\left\{\begin{array}{l}
y-a x^{3}+b x^{2}-z+I=0, \\
c-d x^{2}-y=0 .
\end{array}\right.
$$

The plot of the slow curve is presented on Fig. 1. 


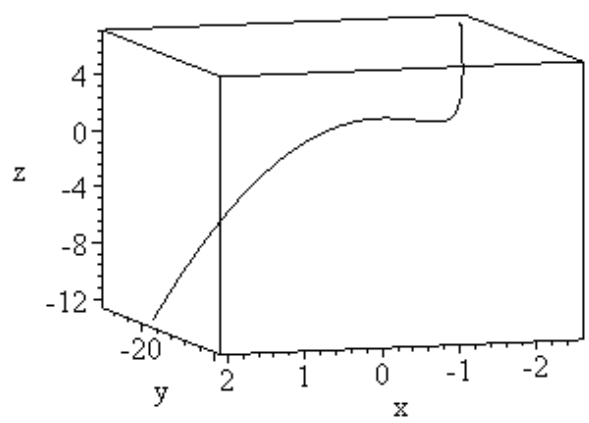

Fig. 1. The slow curve (7) of the system (6); $a=1, b=3, c=1, d=5, I=2.7$

From (7) we get

$a x^{3}+(d-b) x^{2}+z-I-c=0$,

which allow us to investigate a projection of the slow curve on the XOZ-plane, see Fig.2.

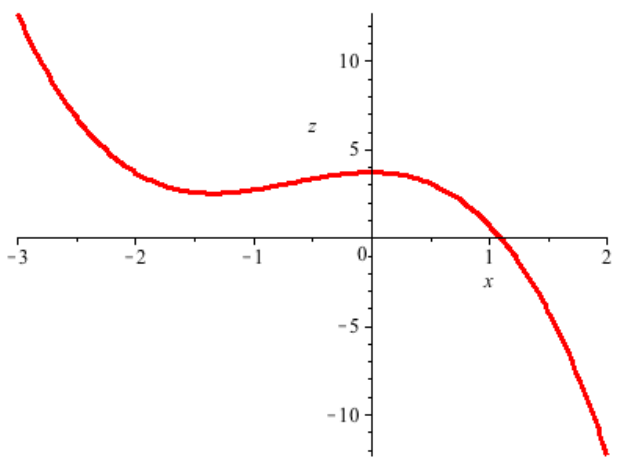

Fig. 2. The projection of the slow curve on the XOZ-plane. The parameters' values are the same as for Fig. 1

The Jacobian matrix (3) for the system (6)

$J=\left(\begin{array}{cc}-3 a x^{2}+2 b x & 1 \\ -2 d x & -1\end{array}\right)$

has a characteristic equation

$\lambda^{2}+\lambda\left(1+3 a x^{2}-2 b x\right)+3 a x^{2}+2(d-b) x=0$.

The necessary condition of stability (which is also the sufficient in this case) for the polynomial (8) is 


$$
\left\{\begin{array}{l}
1+3 a x^{2}-2 b x>0, \\
(3 a x+2 d-2 b) x>0 .
\end{array}\right.
$$

From (9) we can determine the abscissas of the points of an expected change of stability of the slow curve:

$$
x_{1}=\frac{2(b-d)}{3 a}, \quad x_{2}=0, \quad x_{3,4}=\frac{b \pm \sqrt{b^{2}-3 a}}{3 a} .
$$

These points divide the slow curve into several parts, see Fig. 3.

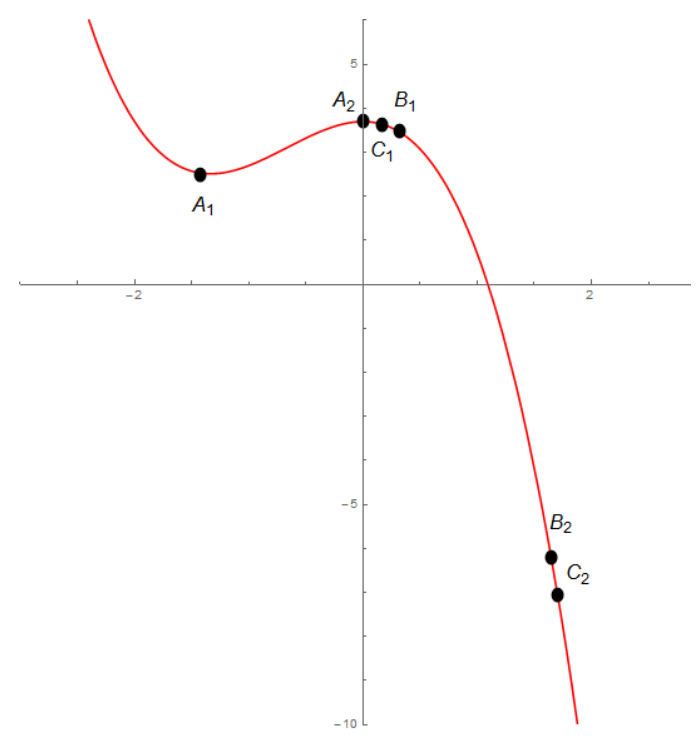

Fig. 3. The projection of the slow curve on the XOZ-plane and the points of changing of stability

We check the sign of real part of eigenvalues of the matrix $J$ for all parts to find out whether the region is stable or unstable. One of the two real eigenvalues of the Jacobian matrix of the fast system changes its sign at the points $A_{1}$ and $A_{2}$, and at these points the slow curve changes its stability. Thus, $A_{1}$ and $A_{2}$ are the jump points. The proper choice of an additional parameter of the system allows us to glue the stable and the unstable integral manifolds of the system that exist in the $\varepsilon$-neighborhood of the stable and unstable regions of the slow curve. As a result of this gluing we obtain a canard.

However, there are other points of the stability's change (see $B_{1}$ and $B_{2}$ on Fig. 3), which are not jump points, because the trajectory does not immediately escape the slow integral manifold as soon as it reaches these points, compared to the previous case. One can find them by equating the real parts of the complex conjugate eigenvalues of $J$ 


$$
\lambda=\frac{-\left(1+3 a x^{2}-2 b x\right) \pm \sqrt{\left(1+3 a x^{2}-2 b x\right)^{2}-4\left(3 a x^{2}+2 d x-2 b x\right)}}{2}
$$

to zero. The phenomenon of the delay of loss of stability occurs as the trajectory goes through these points.

There are points $C_{1}$ (between points $A_{2}$ and $B_{1}$ ) and $C_{2}$ (to the right from the point $B_{2}$ ) on the slow curve, at which the pair of real eigenvalues of the Jacobian matrix $J$ becomes the pair of the complex conjugate eigenvalues, see Fig. 3 .

From (10) it is possible to find the relations between parameters values

$$
d=\frac{3 a}{2\left(b+\sqrt{b^{2}-3 a}\right)},
$$

under which the points $A_{2}, B_{1}$ and $C_{1}$ coincide. In that case the real and the imaginary parts of the pair of complex conjugate eigenvalues become equal to zero simultaneously, and a multiple zero root arises with the following emergence of the pair of real eigenvalues with the opposite sign.

Under condition (11) and the proper choice of the values of the parameters it is possible to construct the slow integral manifold with multiple change of stability: we need one parameter, say $s$, to glue the stable and unstable slow integral manifolds at the point $A_{1}$ using the techniques of canards (see Figs. 4 and 5), and we need two more parameters, say $a$ and $I$, for gluing integral manifolds at the point $A_{2}$ with help the method described in $[22,23]$.

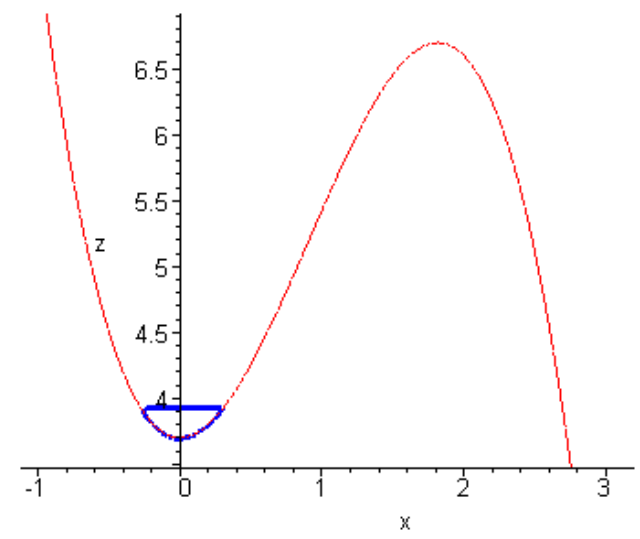

Fig. 4. The projections of the canard without head (blue line) and the slow curve (red line) of the system (6) under the condition (11) and $a=1, b=3, c=1, \alpha=-1.2, I=2.7, \varepsilon=0.01$, $s=3.0810445478558141214$ 


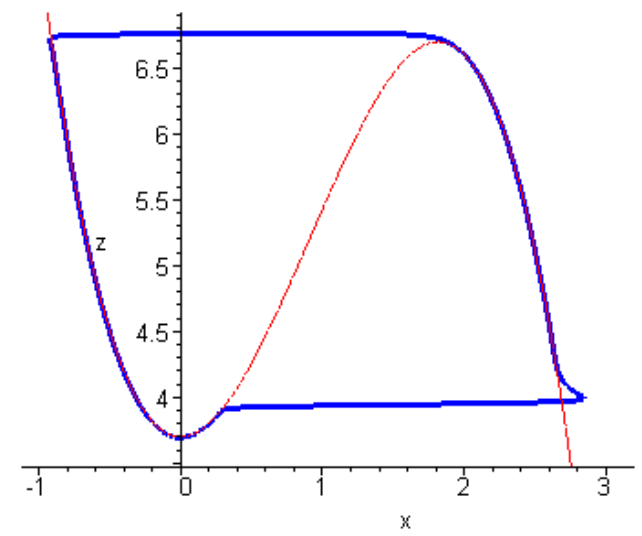

Fig. 5. The projections of the canard with head (blue line) and the slow curve (red line) of the system (6) under the condition (11) and $a=1, b=3, c=1, \alpha=-1.2, I=2.7, \varepsilon=0.01$,

$$
s=3.0810445478558141213
$$

As result of these gluing procedures we get the slow integral manifold with multiple change of stability (see Fig. 6) that looks like a canard cascade [24]. The difference between these two objects consists in that for a canard cascade we apply the canard technique only.

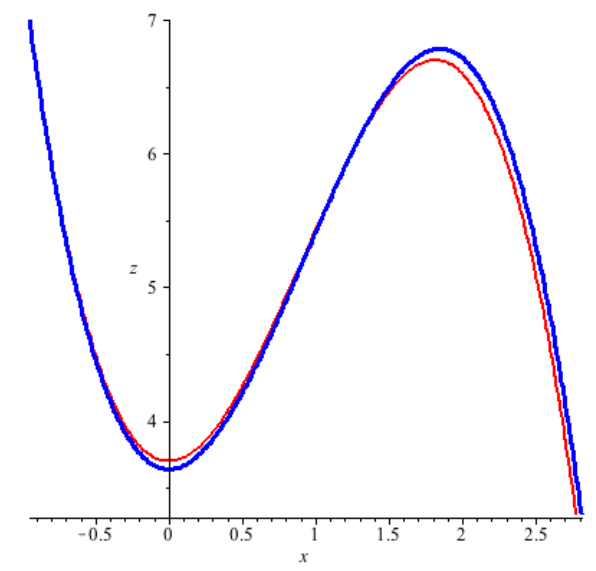

Fig. 6. The projections of the integral manifold with multiple change of stability (blue line) and the slow curve (red line) of the system (6)

\section{Conclusion}

In this paper we investigated the different scenarios of the changing of stability of slow integral manifold via the Hindmarsh-Rose model. We have shown that in this singularly perturbed system the equilibrium of the fast subsystem loses its stability with the passage of one or a pair of complex conjugate eigenvalues through the imaginary axis when the slow variables are changed. 
Moreover, it was shown that there is the scenario of change of stability when the real parts as well as the imaginary parts of a pair of complex conjugate eigenvalues become zero followed by the appearance of multiple zero root and then by the birth of a pair of real eigenvalues of opposite signs. The crucial result of present investigation is that it is possible to construct the slow integral manifold with multiple change of its stability for some values of the additional parameters of the singularly perturbed system.

\section{Acknowledgment}

This work is supported in part by the Russian Foundation for Basic Research (grant 14-01-97018-p) and the Ministry of Education and Science of the Russian Federation under the Competitiveness Enhancement Program of Samara University (2013-2020).

\section{References}

1. Shchepakina E, Sobolev V. Black swans and canards in laser and combustion models. Chapter 8 (51 p.) in "Singular Perturbations and Hysteresis", ed. by M.P. Mortell, R. O’Malley, A. Pokrovskii, V. Sobolev. Philadelphia, SIAM, 2005.

2. Shchepakina E, Sobolev V, Mortell MP. Singular Perturbations: Introduction to System Order Reduction Methods with Applications. In: Springer Lecture Notes in Mathematics, Vol. 2114. Cham: Springer International Publishing, 2014.

3. Kononenko LI, Sobolev VA. Asymptotic expansion of slow integral manifolds. Sib. Math. J., 1994; 35: 1119-1132.

4. Arnold VI, Afraimovich VS, Il'yashenko YuS, Shil'niko LP. Theory of bifurcations. In "Dynamical Systems", ed. by V. Arnold, Encyclopedia of Mathematical Sciences. New York, Springer, 1994; 5.

5. Shchepakina EA. Two forms of stability change for integral manifolds. Differential Equations, 2004; 40(5): 766-769.

6. Benoit E, Calot JL, Diener F, Diener M. Chasse au canard [The duck shooting]. Collectanea Mathematica, 1981; 31-32: 37-119. [in French]

7. Gorelov GN, Sobolev VA. Mathematical modelling of critical phenomena in thermal explosion thory. Combust. Flame, 1991; 87: 203-210.

8. Gorelov GN, Sobolev VA. Duck-trajectories in a thermal explosion problem. Appl. Math. Lett., 1992; 5(6): 3-6.

9. Sobolev VA, Shchepakina EA. Duck trajectories in a problem of combustion theory. Differential Equations, 1996; 32: 1177-1186.

10.Shchepakina EA. Black swans and canards in self-ignition problem. Nonlinear Anal. Real World Appl., 2003; 4(1): 45-50.

11.Shchepakina E. Canards and black swans in model of a 3-D autocatalator. Journal of Physics: Conference Series, 2005; 22: 194-207.

12.Shchepakina E, Korotkova O. Condition for canard explosion in a semiconductor optical amplifier. Journal of the Optical Society of America B: Optical Physics, 2011; 28(8): 1988-1993.

13.Pokrovskii A, Rachinskii D, Sobolev V, Zhezherun A. Topological degree in analysis of cnard-type trajectories in 3-D systems. Applicable Analysis, 2011; 90(7): 1123-1139.

14.Shchepakina E, Korotkova O. Canard explosion in chemical and optical systems. Discrete and Continuous Dynamical Systems - Series B, 2013; 18(2): 495-512. 
15.Shchepakina EA. Critical phenomena in a model of fuel's heating in a porous medium. CEUR Workshop Proceedings, 2015; 1490: 179-189. DOI: 10.18287/1613-0073-20151490-179-189.

16.Sobolev VA. Canards and the effect of apparent disappearance. CEUR Workshop Proceedings, 2015; 1490: 190-197. DOI: 10.18287/1613- 0073-2015-1490-190-197.

17.Shishkova MA. Investigation of a system of differential equations with a small parameter in the highest derivatives. Dokl. Akad. Nauk SSSR, 1973; 209(3): 576-579. [in Russian]; Sov. Math. Dokl., 1973; 14: 483-487. [English transl.]

18.Neustadt AI. Asymptotic investigation of the loss of stability by an equilibrium as a pair of eigenvalues slowly cross the imaginary axis. Usp. Math. Nauk, 1985; 40: 190-191.

19.Neustadt AI. On delayed stability loss under dynamical bifurcation. I, II. Differential Equations, 1987; 23: 2060-2067; 1988; 24: 226-233.

20.Hindmarsh JL, Rose RM. A model of neuronal bursting using three coupled first order differential equations. Proc. R. Soc. London, Ser. B, 1984; 221: 87-102.

21.Hodgkin AL, Huxley AF. A quantitative description of membrane current and its application conduction and excitation in nerve. J. Physiol., 1952; 117(4): 500-544.

22.Shchetinina EV. One task of changing the stability of integrated manifolds. Proceedings of the Academy of Natural Sciences. MMMIU Series, 1999; 3(3): 129-134. [in Russian]

23.Schneider KR, Shchetinina E, Sobolev VA. Control of integral manifolds loosing their attractivity in time. J. Math. Anal. Appl., 2006; 315(2): 740-757.

24.Sobolev V. Canard Cascades. Discrete and Continuous Dynamical Systems. Series B, 2013; 18(3): 513-521. 